\title{
Diode-pumped Yb:CALGO laser with conical refraction output
}

Reza, Md. A., Akbari, R., Fedorova, K., Sokolovskii, G., Rafailov, E., et al.

Md. A. R. Reza, R. Akbari, K. A. Fedorova, G. S. Sokolovskii, E. U. Rafailov, A. Major, "Diode-pumped Yb:CALGO laser with conical refraction output," Proc. SPIE 11259, Solid State Lasers XXIX: Technology and Devices, 112590G (21 February 2020); doi: 10.1117/12.2542077

SPIE. Event: SPIE LASE, 2020, San Francisco, California, United States 


\title{
Diode-pumped Yb:CALGO laser with conical refraction output
}

\author{
Md.A.R. Reza ${ }^{1}$, R. Akbari ${ }^{1}$, K.A. Fedorova ${ }^{2}$, G.S. Sokolovskii ${ }^{3,4}$, E.U. Rafailov ${ }^{5,6}$, and A. Major ${ }^{1}$ \\ ${ }^{1}$ Department of Electrical and Computer Engineering, University of Manitoba, Winnipeg, R3T \\ 5V6, Canada \\ ${ }^{2}$ Department of Physics, Philipps-Universität Marburg, Marburg, 35032, Germany \\ ${ }^{3}$ Ioffe Institute, St. Petersburg, 194021, Russia \\ ${ }^{4}$ Saint Petersburg Electrotechnical University (LETI), St. Petersburg, 197022, Russia \\ ${ }^{5}$ School of Engineering \& Applied Science, Aston University, Birmingham, B4 7ET, UK \\ ${ }^{6}$ Saratov State University, Astrakhanskaya Str. 83, Saratov 410012, Russia
}

\begin{abstract}
A high power conical refraction (CR) laser was demonstrated based on Yb:CALGO laser crystal with a separate intracavity CR element. The CR laser delivered the maximum output power of $6.25 \mathrm{~W}$ at $25 \mathrm{~W}$ of incident pump power which is the highest output power for the CR lasers to date. The separation of the CR element from the laser gain medium reduced the complexity of laser pumping. The generated CR laser beam exhibited excellent quality with well-resolved concentric rings and the Poggendorff dark ring.
\end{abstract}

Keywords: Conical refraction, Yb-ion lasers, diode-pumped lasers.

\section{INTRODUCTION}

The generation of CR laser beam has recently attracted a lot of attention due to its potential applications for microscopy, optical trapping, communication, etc. [1]. In a uniaxial crystal with two principal refractive indices, the passing of a light beam through the crystal shows birefringence, i.e. it yields two rays called ordinary ray and extraordinary ray. The extraordinary ray lies with an angle with respect to the ordinary ray if the input beam is not propagating along the optical axis. In a biaxial crystal with three principal refractive indices and two optical axes, light propagation along the optical axes is a bit different from the uniaxial crystal. In a biaxial crystal, when light enters along the optical axis, one can observe a hollow cylinder in the output with annular intensity profile after the beam propagate conically through the crystal $[1,2]$. Using aragonite and sunlight, Lloyd observed the transformation of the double refraction into CR light when the optical axis of the crystal was aligned with the incident beam [3]. Although Lloyd observed the CR phenomenon, but it was Poggendorf who demonstrated the first CR beam with excellent quality, i.e. the light rings of the CR beam were separated by a dark ring [4]. In 1941 Raman published his works [57] based on naphthalene crystals which are biaxial crystals with 10 times larger birefringence when compared to aragonite. In his works, he observed the Poggendorf dark ring between the two bright CR rings. In addition to that, he also recorded the variation in the intensity of the CR ring along the beam propagation path. He found that in the farfield the CR beam with Poggendorff dark ring between the two bright CR rings collapsed into a bright spot which is known as Raman spot [5-7].

The theory of the formation of the CR laser light and its properties can be understood from previous works [8-13]. In some of the previous reports [14-18], the laser gain medium also worked as a CR element (CRE). It complicates the understanding of the effect of the CR on the Gaussian laser mode. Recently, a new set up for CR laser was proposed where the CRE was kept separate from the laser crystal [19,20]. Keeping the CRE separate from the laser crystal does not only allow the power scaling but it also allows for laser adjustment to achieve better conditions of CR. Also, in this separate arrangement, the laser crystal properties are independent of the CR crystal properties. 
Yb-ion lasers [21-23] are very popular because of their simple electronic configuration, ${ }^{2} \mathrm{~F}_{7 / 2}$ - the ground manifold and ${ }^{2} \mathrm{~F}_{5 / 2}$ - the upper manifold. As Yb-ion lasers do not have multiple higher levels, the effects such as up-conversion, concentration quenching, excited-state absorption are absent. Also, since the quantum defect (the difference between the pump and laser photon energies) is low this makes $\mathrm{Yb}$-ion crystals attractive for high power laser operation [2431]. Considering these important characteristics, Yb-ion lasers are becoming reliable in applications such as micromachining [32], optical communication [33], multiphoton microscopy [34-39] and nonlinear optics [40-43]. There are different types of $\mathrm{Yb}$ laser crystals available but $\mathrm{Yb}$ :CALGO is the most popular one because of its high thermal conductivity $(6.5 \mathrm{~W} / \mathrm{m} / \mathrm{K})$ with a $50 \mathrm{~nm}$ broad emission spectrum which makes it suitable for high power laser operation [44-55].

In this work, a multi-watt power scalable CR laser was investigated by using Yb:CALGO as the laser host and a separate intracavity CR element. An output power of $6.25 \mathrm{~W}$ was obtained for $25 \mathrm{~W}$ of incident pump power. We also investigated the beam evolution in the Lloyd image plane. We were able to generate a high quality CR laser beam with clear Poggendorff dark ring.

\section{EXPERIMENTAL SETUP}

A 5 mm-long Yb:CALGO crystal ( $a$-cut,2 at. \%) was used in a five-mirror cavity configuration. Both input surfaces of the crystal had antireflection coating for pump and laser wavelengths. The cavity is shown in figure 1 .

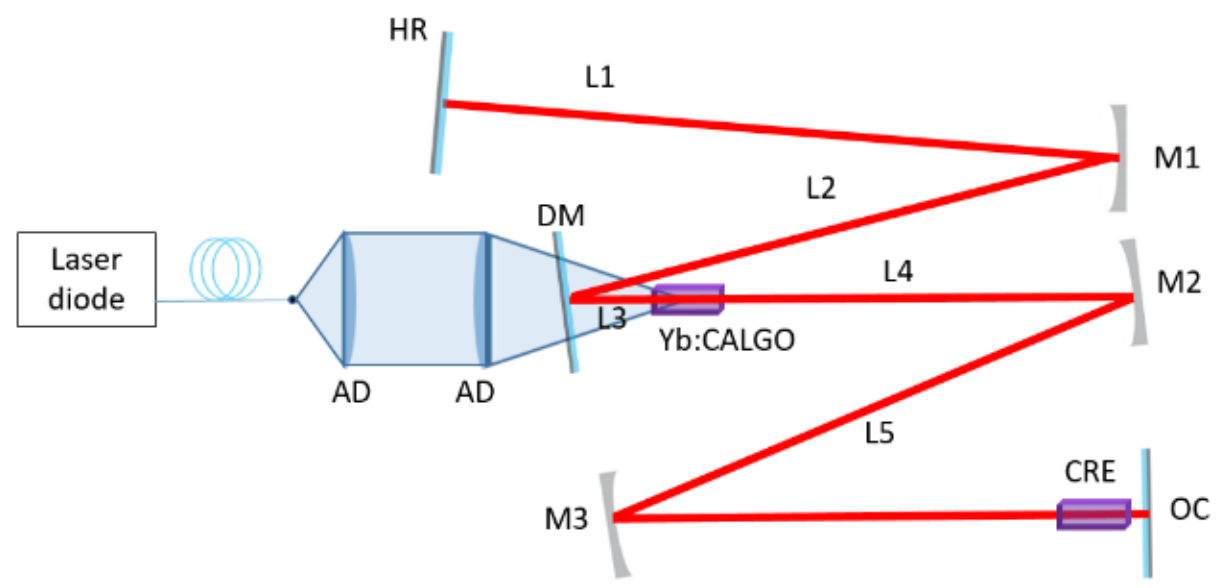

Fig. 1. Schematic of the laser cavity. M1 $=-300 \mathrm{~mm}, \mathrm{M} 2=-250 \mathrm{~mm}, \mathrm{M} 3=-150 \mathrm{~mm}, \mathrm{OC}$ - output coupler, HR - highly reflective mirror, CRE - CR element.

The fiber-coupled laser diode delivered an unpolarized laser beam at $979 \mathrm{~nm}$ (N.A. $=0.22$, fiber core diameter $=105$ $\mu \mathrm{m})$ and was imaged into a spot size diameter of $157 \mu \mathrm{m}$ in the laser crystal by using two achromatic doublet lenses. The laser cavity was optimized for the non-CR regime of operation (i.e. without a CR crystal in the cavity) by compensating for the thermal effect and optimizing the mode matching between the cavity mode and the pump beam. The maximum absorbed pump power under non-lasing condition was $13.22 \mathrm{~W}$ at an incident pump power of $25 \mathrm{~W}$. The highest output power was $7.47 \mathrm{~W}$ at $25 \mathrm{~W}$ of incident pump power with $10 \% \mathrm{OC}$ (output coupler).

After characterizing the laser in the continuous-wave (CW) regime, an $18 \mathrm{~mm}$-long KGW crystal cut along its optical axis (the CR crystal) was placed between the focusing mirror M3 and the output coupler to observe CR laser radiation. The KGW crystal was also used in the previous experiments as a CRE [14-20,56-58]. The reason for the popularity of the KGW is the strong anisotropy in its optical and physical properties [59-60] as well as high optical quality. The latter also made it a popular gain medium for diode-pumped lasers in the $\mathrm{CW}$ and pulsed regimes [61-66]. The radius of curvature (ROC) of the focusing mirror M3 has a strong effect on the quality of the generated CR beam and the 
details have been discussed in Ref. 19. Although increasing the ROC of M3 gives slightly higher output power, the pattern of the CR beam loses its double-ring structure. So, the lower ROC of M3 is a good choice considering the good CR beam quality with decent output power [19]. In our case, we chose M3 $=-150 \mathrm{~mm}$ which yielded a very high quality $\mathrm{CR}$ beam with $6.25 \mathrm{~W}$ of output power at $25 \mathrm{~W}$ of incident pump power.

\section{RESULTS AND DISCUSSION}

To generate a CR output beam, the optical axis of the CRE and the laser mode axis should be in parallel with each other. So, the alignment of the CRE was the most challenging part of the experiment. In the initial stage of the alignment, negligible output power indicated a large misalignment between the optical axis of the CRE and the laser mode axis. Further adjustment of the CR crystal resulted in a doubly refracted laser output with increased output power. At this stage, we needed to align the CR crystal together with the output coupler to obtain the CR pattern. We believe that a nice CR beam with a dark Poggendorff ring was obtained for perfect parallel orientation of the CR crystal along the cavity axis. The output beam was observed with a $60 \mathrm{~mm}$ imaging lens and a CCD camera.

The CR laser delivered a maximum output power of $6.25 \mathrm{~W}$ at $25 \mathrm{~W}$ of incident pump power by using a $10 \%$ output coupler. Using the lens and CCD camera, we recorded the evolution of CR beam intensity pattern after the output coupler. The position of the image plane was 1-3 mm away from the OC. As we got away from the Lloyd image plane along the beam propagation axis, the distinguishing features of the beam became faded and finally far away from the image plane we observed the Raman spot. The beam evolution is shown in figures 2(a)-(f). Another unique property of the CR laser beam is the polarization state distribution along with the CR pattern where every two diametrically opposite points have orthogonal polarization. The polarization state distribution along the $\mathrm{CR}$ beam was examined by using a polarizing beam splitter and indicated in figure 2(f).

(a)

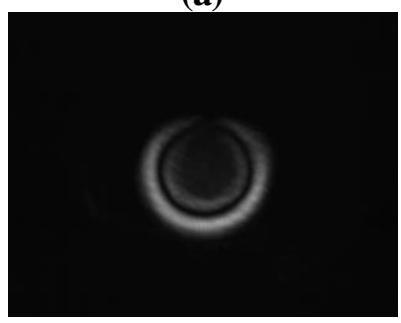

(d)

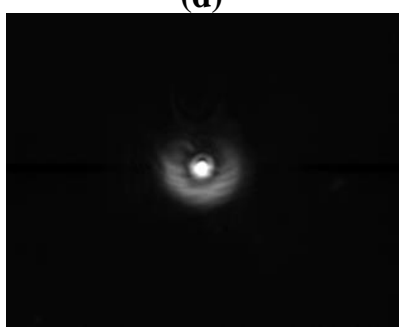

(b)

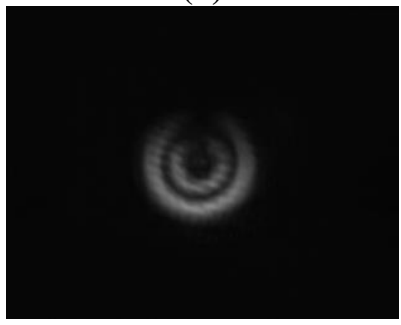

(e)

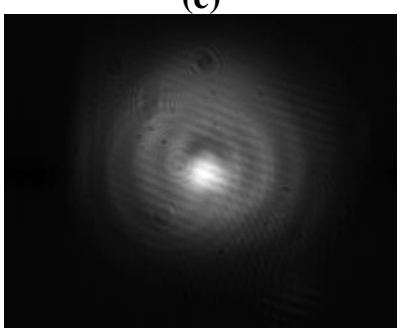

(c)

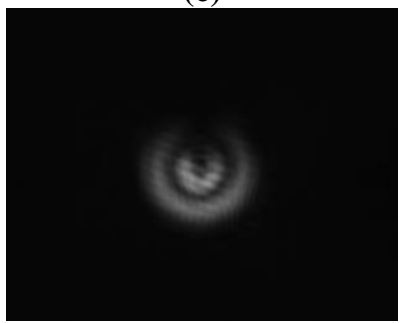

(f)

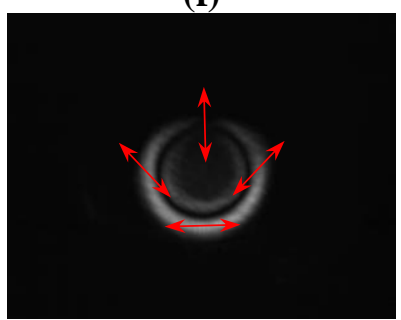

Fig. 2. The CR laser beam at the Lloyd image plane and its free-space evolution for the CRE with a horizontal orientation of its $\mathrm{N}_{\mathrm{m}}$-axis. It is mention-worthy that $\mathrm{z}$ is measured from the front surface of the OC: (a) $\mathrm{z}=6.7 \mathrm{~mm}$, (b) $26.4 \mathrm{~mm}$, (d) $41.7 \mathrm{~mm}$, (e) $73.64 \mathrm{~mm},(\mathrm{~g})$ is $\mathrm{z}=300 \mathrm{~mm}$ which is far filed view of the beam. (f) Local polarization states determined by a polarizer.

An important parameter that determines the quality of the CR output beam is the dimensionless parameter $\rho_{0}$, defined as the ratio of the radius of the CR beam to the input beam radius $\rho_{0}=\mathrm{R}_{0} / \mathrm{W}_{0}$ [1]. To have a clear CR beam with distinguishing Poggendorff dark ring, the recommended value is $\rho_{0}>>1$. In our case, $\rho_{0}$ was approximately 4 owing to the choice of small ROC of the focusing mirror M3. 
To compare the performance of the CR laser against an equivalent non-CR laser (i.e. a typical laser with a Gaussian beam intensity profile), the CRE was replaced by a $20 \mathrm{~mm}$-long AR-coated $\mathrm{N}_{\mathrm{g}}$-cut (non-CR) $\mathrm{KGW}$ crystal in the cavity. With a $10 \%$ output coupler, the maximum output power obtained with the undoped non-CR KGW crystal was $7.21 \mathrm{~W}$ at $25 \mathrm{~W}$ of pump power. In figure 3 the comparison of the output power among the $\mathrm{CW}$, non-CR laser with the undoped $\mathrm{KGW}$ and the $\mathrm{CR}$ laser is given with respect to the corresponding input pump power.

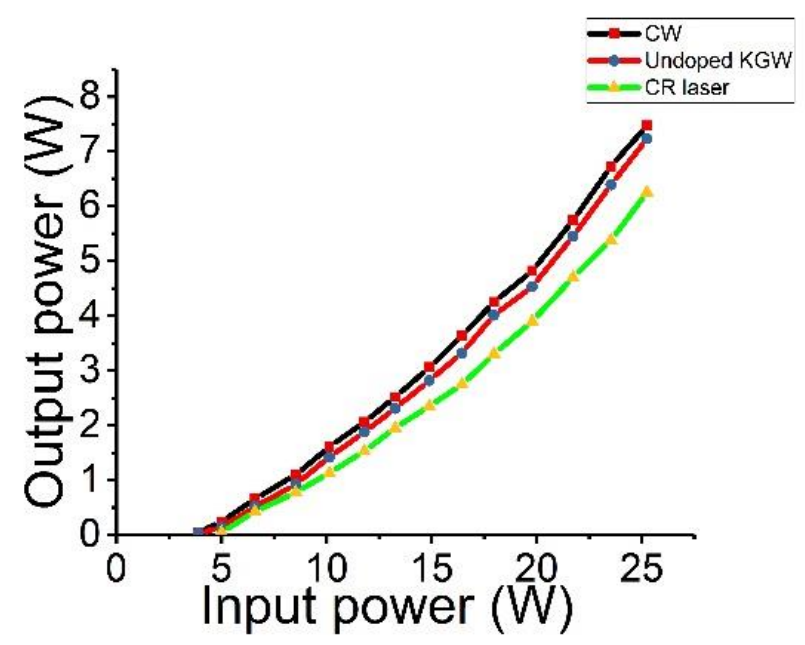

Fig. 3. The output powers vs. the incident pump power for the free-running CW, non-CR and CR lasers.

\section{CONCLUSION}

In conclusion, a high power Yb:CALGO laser with a well-characterized CR beam based on a separate intracavity CR element was demonstrated. The power scalability of our laser was facilitated by the separation of the laser host and CR element. Because of this approach the CR beam properties became more independent from the lasing properties of the laser host (e.g. wavelength) and the cavity design parameters (e.g. pump spot size). The laser delivered a maximum output power of $6.25 \mathrm{~W}$ at $25 \mathrm{~W}$ of incident pump power. In addition to that, the beam evolution pattern was also observed. At the Lloyd image plane a clear CR beam with two concentric rings separated by the Poggendorff dark ring was obtained which in the far-field became a spot known as Raman. Future experiments will focus on further power scaling including the use of popular Nd-ion laser crystals [67-70] as well as continuous or discrete wavelength tunability [71-75] of the produced CR radiation.

\section{ACKNOWLEDGEMENTS}

The authors would like to acknowledge funding of this project provided by the Natural Science and Engineering Research Council of Canada (NSERC), Western Economic Diversification Canada, and the University of Manitoba. E.U.R was partially supported by a Grant of Russian Science Foundation (Grant No. 18-15-00172).

\section{REFERENCES}

[1] A. Turpin, Y. V. Loiko, T. K. Kalkandjiev, and J. Mompart, "Conical refraction: fundamentals and applications," Laser Photon. Rev. 10, 750-771 (2016).

[2] W. R. Hamilton, "Third supplement to an essay on the theory of systems of rays," Trans. R. Irish Acad. 17, 1 (1837). 
[3] H. Lloyd, "On the phenomena presented by light in its passage along the axes of biaxal crystals," Philos. Mag. 2, $112(1833)$.

[4] J. C. Poggendorff, "Ueber die konische refraction,"Ann. Phys. Chem. 124, 461 (1839).

[5] C. V. Raman, V. S. Rajagopalan, and T. M. K. Nedungadi, "Conical refraction in naphthalene crystals," Nature 147, 268 (1941).

[6] C. V. Raman, V. S. Rajagopalan, and T. M. K. Nedungadi, "Conical refraction in naphthalene crystals," Proc. Indian Acad. Sci. 14, 221 (1941).

[7] C. V. Raman, "The phenomena of conical refraction," Curr. Sci. 11, 46 (1942).

[8] C. F. Phelan, K. E. Ballantine, P. R. Eastham, J. F. Donegan, and J. G. Lunney, "Conical diffraction of a Gaussian beam with a two-crystal cascade," Opt. Express 20, 13201 (2012).

[9] G. S. Sokolovskii, D. J. Carnegie, T. K. Kalkandjiev, and E. U. Rafailov, "Conical Refraction: New observations and a dual cone model," Opt. Express 21, 11125 (2013).

[10] M. V. Berry, "Conical diffraction asymptotics: fine structure of Poggendorff rings and axial spike," J. Opt. A 6, 289 (2004).

[11] A. M. Belsky and M. A. Stepanov, "Internal conical refraction of coherent light beams," Opt. Commun. 167, 1 (1999).

[12] M. V. Berry, M. R. Jeffrey, and J. G. Lunney, "Conical diffraction: Observations and theory," Proc. R. Soc. A 462, 1629 (2006).

[13] A. Turpin, Y. V. Loiko, T. K. Kalkandjiev, H. Tomizawa, and J. Mompart, "Super-Gaussian conical refraction beam," Opt. Lett. 39, 4349 (2014).

[14] R. Cattoor, I. Manek-Hönninger, D. Rytz, L. Canioni, and M. Eichhorn, "Laser action along and near the optic axis of a holmium-doped $\mathrm{KY}\left(\mathrm{WO}_{4}\right)_{2}$ crystal," Opt. Lett. 39, 6407 (2014).

[15] K. G. Wilcox, A. Abdolvand, T. K. Kalkandjiev, and E. U. Rafailov, "Laser with simultaneous Gaussian and conical refraction outputs," Appl. Phys. B 99, 619 (2010).

[16] J. Hellström, H. Henricsson, V. Pasiskevicius, U. Bünting, and D. Haussmann, "Polarization-tunable Yb:KGW laser based on internal conical refraction," Opt. Lett. 32, 2783 (2007).

[17] A. Brenier, "Lasing with conical diffraction feature in the KGd(WO4)2:Nd biaxial crystal," Appl. Phys. B 122, 237 (2016).

[18] A. Abdolvand, K. G. Wilcox, T. K. Kalkandjiev, and E. U. Rafailov, "Conical refraction Nd:KGd($\left(\mathrm{WO}_{4}\right)_{2}$ laser," Opt. Express 18, 2753 (2010).

[19] R. Akbari, C. Howlader,1 K. A. Fedrova, G. S. Sokolovoskii, E. U. Rafailov and A. Major, "Conical refraction output from a Nd: $\mathrm{YVO}_{4}$ with an intracavity conerefringent element," Opt. Lett. 44(3), 642 (2019).

[20] C. Howlader, M. Nadimi, T. Waritanant, K.A. Fedorova, G.S. Sokolovskii, E.U. Rafailov, A. Major, "High power Nd:YVO-KGW conical refraction laser," Proc. SPIE 10904, 109041X (2019).

[21] L. DeLoach, S. Payne, L. Chase, L. Smith, W. Kway, W. Krupke, "Evaluation of absorption and emission properties of $\mathrm{Yb}^{3+-}$ doped crystals for laser applications," IEEE J. Quant. Electron. 29, 1179-1191(1984).

[22] W. Krupke, "Ytterbium solid-state lasers - the first decade," IEEE J. Sel. Top. Quantum Electron. 6(6), 1287$1296(2000)$.

[23] F. Druon, F. Balembois, and P. Georges, "New laser crystals for the generation of ultrashort pulses," Comptes Rendus Physique 8, 153-164 (2007).

[24] T. Südmeyer, S. V. Marchese, S. Hashimoto, C. R. E. Baer, G. Gingras, B. Witzel, U. Keller, "Femtosecond laser oscillators for high-field science," Nat. Photon. 2(10), 599-604 (2008).

[25] H. Zhao, A. Major, "A continuous wave Yb:KGW laser with polarization-independent pump absorption," Laser Phys. 23, 095001 (2013).

[26] H. Zhao, A. Major, "Orthogonally polarized dual-wavelength Yb:KGW laser induced by thermal lensing," Appl. Phys. B 122(6), 163-169 (2016).

[27] R. Akbari, H. Zhao, and A. Major, "High-power continuous-wave dual-wavelength operation of a diodepumped Yb:KGW laser," Opt. Lett. 41(7), 1601-1604 (2016).

[28] A. Major, D. Sandkuijl, V. Barzda, "A diode-pumped continuous-wave Yb:KGW laser with Ng-axis polarized output," Laser Phys. Lett. 6(11), 779-781 (2009).

[29] S. Manjooran, A. Major, "Efficient operation of a diode-pumped multi-watt continuous wave Yb:KYW laser with excellent beam quality," Laser Phys. Lett. 14(3), 035805 (2017).

[30] R. Akbari, K. A. Fedorova, E. U. Rafailov and A. Major, "Diode-pumped ultrafast Yb:KGW laser with $56 \mathrm{fs}$ pulses and multi-100 kW peak power based on SESAM and Kerr-lens mode locking," Appl. Phys. B 123, 123(2017). 
[31] R. Akbari and A. Major, "High power diode-pumped Kerr-lens mode locked bulk Yb:KGW laser," Appl. Opt. 56, 8838 (2017).

[32] R. R. Gattass and E. Mazur, "Femtosecond laser micromachining in transparent materials," Nat. Photon. 2(4), 219-225 (2008).

[33] A. M. Weiner, Ultrafast Optics, John Wiley \& Sons, 2009.

[34] W. R. Zipfel, R. M. Williams, and W. W. Webb, "Nonlinear magic: multiphoton microscopy in the biosciences," Nat. Biotechnol. 21, 1369-1377 (2003).

[35] C. Xu and F. W. Wise, "Recent advances in fibre lasers for nonlinear microscopy," Nat. Photon. 7(11), 875882 (2013).

[36] N. Prent, C. Green, C. Greenhalgh, R. Cisek, A. Major, B. Stewart, and V. Barzda, "Inter-myofilament dynamics of myocytes revealed by second harmonic generation microscopy," J. Biomed. Opt. 13(4), 041318 (2008).

[37] A. Major, R. Cisek and V. Barzda, "Development of diode-pumped high average power continuous-wave and ultrashort pulse Yb:KGW lasers for nonlinear microscopy," Proc. SPIE 6108, 61080Y (2006).

[38] A. Major, R. Cisek, A. Tuer, N. Prent, V. Barzda, "Ultrafast $\mathrm{Yb}: \mathrm{KGd}\left(\mathrm{WO}_{4}\right)_{2}$ laser for multimodal biomedical imaging with reduced photodamage," Proc. SPIE 6881, 688108 (2008).

[39] L.B. Mostaço-Guidolin, A.C. Ko, D.P. Popescu, M.S. Smith, E.K. Kohlenberg, M. Shiomi, A. Major, and M.G. Sowa, "Evaluation of texture parameters for the quantitative description of multimodal nonlinear optical images from atherosclerotic rabbit arteries," Phys. Med. Biol. 56(16), 5319-5334 (2011).

[40] S. Manjooran, H. Zhao, I. T. Lima Jr., and A. Major, "Phase-matching properties of PPKTP, MgO:PPSLT and MgO:PPcLN for ultrafast optical parametric oscillation in the visible and near-infrared ranges with green pump," Laser Phys. 22(8), 1325-1330 (2012).

[41] H. Zhao, I. T. Lima Jr., and A. Major, "Near-infrared properties of periodically poled $\mathrm{KTiOPO}_{4}$ and stoichiometric MgO-doped $\mathrm{LiTaO}_{3}$ crystals for high power optical parametric oscillation with femtosecond pulses," Laser Phys. 20(6), 1404-1409 (2010).

[42] I.T. Lima Jr., V. Kultavewuti, and A. Major, "Phasematching properties of congruent MgO-doped and undoped periodically poled $\mathrm{LiNbO}_{3}$ for optical parametric oscillation with ultrafast excitation at $1 \mu \mathrm{m}$," Laser Phys. 20(1), 270-275 (2010).

[43] I.P. Nikolakakos, A. Major, J.S. Aitchison and P.W.E. Smith, "Broadband characterization of the nonlinear optical properties of common reference materials," IEEE J. Quantum Electron. 10(5), 1164-1170 (2004).

[44] S. Manjooran and A. Major, "Diode-pumped $45 \mathrm{fs} \mathrm{Yb:CALGO} \mathrm{laser} \mathrm{oscillator} \mathrm{with} 1.7 \mathrm{MW}$ of peak power," Opt. Lett. 43(10), 2324-2327 (2018).

[45] S. Manjooran, P. Loiko, A. Major, "A discretely tunable dual-wavelength multi-watt Yb:CALGO laser," Appl. Phys. B 124(1), 13 (2018).

[46] P. Sévillano, P. Georges, F. Druon, D. Descamps, and E. Cormier, "32-fs Kerr-lens mode-locked Yb: $\mathrm{CaGdAlO}_{4}$ oscillator optically pumped by a bright fiber laser," Opt. Lett. 39(20), 6001-6004 (2014).

[47] A. Diebold, F. Emaury, C. Schriber, M. Golling, C. J. Saraceno, T. Südmeyer, and U. Keller, "SESAM modelocked $\mathrm{Yb}: \mathrm{CaGdAlO}_{4}$ thin disk laser with 62 fs pulse generation," Opt. Lett. 38(19), 3842-3845 (2013).

[48] S. Manjooran, A. Major, "Generation of sub-50 fs pulses with >1.5 MW of peak power from a diode-pumped Yb:CALGO laser oscillator," in Conference on Lasers and Electro-Optics (Optical Society of America, 2016), p. JTu5A.82.

[49] P. Loiko, J. M. Serres, X. Mateos, X. Xu, J. Xu, V. Jambunathan, P. Navratil, A. Lucianetti, T. Mocek, X. Zhang, U. Griebner, V. Petrov, M. Aguiló, F. Díaz, and A. Major, "Microchip Yb:CaLnAlO 4 lasers with up to $91 \%$ slope efficiency," Opt. Lett. 42(13), 2431-2434 (2017).

[50] Y. Zaouter, J. Didierjean, F. Balembois, G. Lucas Leclin, F. Druon, and P. Georges, "47-fs diode-pumped $\mathrm{Yb}^{3+}$ : CaGdAlO 4 laser," Opt. Lett. 31(1),119-121 (2006).

[51] A. Klenner, M. Golling, and U. Keller, "Gigahertz diode-pumped Yb:CALGO laser with 60-fs pulses and an average output power of 3.5 W", in Conference on Lasers and Electro-Optics (Optical Society of America, 2014), p. SM4F.6.

[52] P. Sevillano, R. Dubrasquet, P. Georges, F. Druon, D. Descapms and E. Cormier, “37fs-1.5W Kerr-lens modelocked Yb:CALGO laser oscillator," in Conference on Lasers and Electro-Optics (Optical Society of America, 2014), p. STu2E.1.

[53] D. N. Papadopoulos, F. Druon, J. Boudeile, I. Martial, M. Hanna, P. Georges, P. O. Petit, P. Goldner, and B. Viana, "Low-repetition-rate femtosecond operation in extended cavity mode-locked Yb:CALGO laser," Opt. Lett. 34(2), 196-198 (2009). 
[54] A. Calendron, "Dual-crystal Yb:CALGO high power laser and regenerative amplifier," Opt. Express 21, 26174 (2013).

[55] A. Klenner, M. Golling, and U. Keller, "High peak power Gigahertz Yb:CALGO laser," Opt. Express 22, 11125 (2014).

[56] T. K. Kalkandjiev and M. A. Bursukova, "Conical refraction: an experimental introduction,” Proc. SPIE 6994, 69940B (2008).

[57] D. P. O’Dwyer, K. E. Ballantine, C. F. Phelan, J. G. Lunney, and J. F. Donegan, "Optical trapping using cascade conical refraction of light," Opt. Express 20, 21119 (2012).

[58] C. McDonald, C. McDougall, E. Rafailov, and D. McGloin, "Characterizing conical refraction optical tweezers," Opt. Lett. 39, 6691 (2014).

[59] I. V. Mochalov, "Laser and nonlinear properties of potassium gadolinium tungstate laser crystal: $\mathrm{KGd}\left(\mathrm{WO}_{4}\right)_{2} \mathrm{Nd}^{3+}(\mathrm{KGW}: \mathrm{Nd})$," Opt. Eng. 36, 1660 (1997).

[60] P. Loiko, S. Manjooran, K. Yumashev, and A. Major, "Polarization anisotropy of thermal lens in $\mathrm{Yb}: \mathrm{KY}\left(\mathrm{WO}_{4}\right)_{2}$ under high power diode pumping," Appl. Opt. 56, 2937 (2017).

[61] R. C. Talukder, M. Z. E. Halim, T. Waritanant, and A. Major, "Multiwatt continuous wave Nd:KGW laser with hot-band diode pumping," Opt. Lett. 41, 3810 (2016).

[62] M. Z. E. Halim, R. C. Talukder, T. Waritanant, and A. Major, "Passive mode locking of a Nd:KGW laser with hot-band diode pumping," Laser Phys. Lett. 13, 105003 (2016).

[63] R. Akbari and A. Major, "Kerr-lens mode locking of a diode-pumped Yb:KGW laser using an additional intracavity Kerr medium," Laser Phys. Lett. 15(8), 085001 (2018).

[64] R. Akbari and A. Major, "Kerr-lens mode locking of a diode-pumped Yb:KGW laser using an additional intracavity Kerr medium," Laser Phys. Lett. 15(8), 085001 (2018).

[65] A. Major, L. Giniunas, N. Langford, A.I. Ferguson, D. Burns, E. Bente, and R. Danielius, "Saturable Bragg reflector-based continuous-wave mode locking of $\mathrm{Yb}: \mathrm{KGd}\left(\mathrm{WO}_{4}\right)_{2}$ laser," J. Mod. Opt. 49(5), 787-793 (2002).

[66] A. Major, N. Langford, T. Graf and A. I. Ferguson, "Additive-pulse mode locking of a diode-pumped $\mathrm{Nd}: \mathrm{KGd}\left(\mathrm{WO}_{4}\right)_{2}$ laser," Appl. Phys. B 75(4-5), 467-469 (2002).

[67] M. Nadimi, T. Waritanant, and A. Major, "High power and beam quality continuous-wave Nd:GdVO 4 laser in-band diode-pumped at 912 nm," Photon. Res. 5(4), 346-349 (2017).

[68] T. Waritanant, A. Major, "Dual-wavelength operation of a diode-pumped Nd: $\mathrm{YVO}_{4}$ laser at the 1064.1 \& $1073.1 \mathrm{~nm}$ and 1064.1 \& 1085.3 nm wavelength pairs," Appl. Phys. B 124(5), 87 (2018).

[69] T. Waritanant, A. Major, "Diode-pumped Nd: $\mathrm{YVO}_{4}$ laser with discrete multi-wavelength tunability and high efficiency," Opt. Lett. 42, 1149-1152 (2017).

[70] M. Nadimi, T. Waritanant, A. Major, "Discrete multi-wavelength tuning of a continuous wave diode-pumped Nd:GdVO 4 laser," Laser Phys. Lett. 15(5), 055002 (2018).

[71] S. Ghanbari, R. Akbari, and A. Major, "Femtosecond Kerr-lens mode-locked Alexandrite laser," Opt. Express 24(13), 14836-14840 (2016).

[72] S. Ghanbari and A. Major, "High power continuous wave Alexandrite laser with green pump," Laser Phys. 26(7), 75001 (2016).

[73] S. Ghanbari and A. Major, "High power continuous-wave dual-wavelength alexandrite laser," Laser Phys. Lett. 14(10), 105001 (2017).

[74] T. Waritanant, A. Major, "Discretely selectable multiwavelength operation of a semiconductor saturable absorber mirror mode-locked Nd: $\mathrm{YVO}_{4}$ laser," Opt. Lett. 42, 3331-3334 (2017).

[75] M. Kowalczyk, A. Major, and J. Sotor, "High peak power ultrafast $\mathrm{Yb}: \mathrm{CaF}_{2}$ oscillator pumped by a singlemode fiber-coupled laser diode," Opt. Express 25(21), 26289-26295 (2017). 